\title{
Transverse Momentum Dependent Fragmenting Jet Functions with Applications to Quarkonium Production
}

\section{Yiannis Makris*}

Theoretical Division T-2, Los Alamos National Laboratory, Los Alamos, NM, 87545

E-mail: yiannis@lanl.edu

\begin{abstract}
We briefly review the framework of fragmenting jet functions (FJFs) introduced in soft collinear effective theory (SCET) to describe the fragmentation of an identified hadron within a jet. We illustrate how FJFs can be integrated with the leading power (LP) factorization in non-relativistic quantum chromodynamics (NRQCD) in studies of quarkonium production within jets. We present recent theoretical, experimental, and computational developments. We also present the framework of transverse momentum dependent (TMD) FJFs which describe the fragmentation of a hadron within a jet and the transverse momentum of the identified hadron is measured with respect to the jet axis. We show how the measurement of the transverse momentum spectrum can help discriminate between the leading production mechanisms of $J / \psi$.
\end{abstract}

QCD Evolution 2017

22-26 May, 2017

Jefferson Lab Newport News, VA - USA

\footnotetext{
*Speaker.
} 


\section{Quarkonium production in NRQCD}

Quarkonium has been studied widely both experimentally and theoretically and various models have been proposed for describing its properties (such as its decays to light meson and its production processes). Up to date, the most successful approach is that of non-relativistic quantum chromodynamics (NRQCD). NRQCD is an effective field theory (EFT) of QCD which describes non-relativistic dynamics of strong interacting partons. In the case of quarkonium, NRQCD aims to predict the behavior of the heavy quark-antiquark pair within the quarkonium state. Although NRQCD is a very powerful tool for depicting various process involving quarkonium, here we are considering the NRQCD factorization conjecture [1] for quarkonium production.

According to the NRQCD factorization conjecture, the quarkonium production cross section is factorized into the perturbatively calculable short distance coefficients (SDC) and long distance matrix elements (LDME) that need to be extracted from data. More explicitly for the quarkonium state $\mathscr{Q}$ we have,

$$
d \sigma_{N R Q C D}(a+b \rightarrow \mathscr{Q}+X)=\sum_{n} d \sigma(a+b \rightarrow Q \bar{Q}[n]+X)\left\langle\mathscr{O}_{\mathscr{Q}}^{n}\right\rangle,
$$

where

$$
\mathscr{O}_{\mathscr{Q}}^{n}=\mathscr{O}_{2}^{n \dagger}\left(\sum_{J} \sum_{m_{J}}|\mathscr{Q}+X\rangle\langle\mathscr{Q}+X|\right) \mathscr{O}_{2}^{n} \quad \text { and } \mathscr{O}_{2}^{n}=\psi^{\dagger} \mathscr{K}_{n} \chi,
$$

where $\mathscr{K}_{n}$ is a tensor in the Dirac and color algebra and $Q \bar{Q}[n]$ the heavy quark-antiquark pair in particular angular momentum, ${ }^{2 S+1} L_{J}$, and color, $(c)$, configuration (here we use the compact notation, $\left.n={ }^{2 S+1} L_{J}^{(c)}\right)$. While the short distance coefficients $d \sigma(a+b \rightarrow Q \bar{Q}[n]+X)$ in Eq.(1.1) have an expansion in the strong coupling, $\alpha_{s}\left(m_{\mathscr{Q}}\right)$, the LDME scale with the relative velocity of the $Q \bar{Q}$ pair in the quarkonium rest frame, $v$, (see Refs. [2, 3]). Thus truncating the sum can give us the desirable accuracy from a simultaneous expansion in the $\alpha_{s}$ and $v$.

In the quarkonium rest frame the heavy quark-antiquark pair is considered a non-relativistic system, and its kinetic energy is similar to its potential due to the coulomb like forces between the pair. Therefore their relative velocity scales with he strong coupling evaluated at their momentum, $\alpha_{s}\left(m_{Q} v\right)$, and due to asymptotic freedom we have, $v \gtrsim \alpha\left(m_{Q}\right)$. This implies that relativistic corrections in Eq.(1.1) of order $v^{2 n}$ are at least as important as perturbative corrections of order $\alpha\left(m_{Q}\right)^{2 n}$ and therefore reorganization of the perturbative expansion is required in order to include all corrections with similar contributions to the cross section.

Although the NRQCD framework was shown to greatly improve the agreement of theoretical calculations and experimental data, there are many important open questions. In this talk I am particularly interested in the charmonium state $J / \psi$ which is widely studied both experimentally and theoretically but its production mechanism it is yet to be understood. For example, global fit to the worlds data [4] at NLO including the four leading production mechanisms $\left(n=\left\{{ }^{3} S_{1}^{(1)},{ }^{3} S_{1}^{(8)},{ }^{1} S_{0}^{(8)},{ }^{3} P_{J}^{(8)}\right\}\right)$ give LDME which are later used to predict the polarization of the $J / \psi$. The predicted polarization disagrees with measurements of the $J / \psi$ polarization: while the fitted LDME at large $p_{T}$ predict almost transverse polarization, data suggest effectively no polarization $[5,6,7]$. This disagreement is partially attributed to the fact that at large $p_{T}$ fragmentation processes, that for some of the four leading production mechanisms appear first time at NNLO, 


\begin{tabular}{|c|c|c|c|}
\hline Mechanism & LO to $d_{i}^{n}(z)$ & LDME scaling & Total scaling \\
\hline \hline$c \rightarrow\left(c \bar{c}\left[{ }^{3} S_{1}^{(1)}\right]\right)$ & $\mathscr{O}\left(\alpha_{s}^{2}\right)$ & $\sim v^{3}$ & $\sim \alpha_{s}^{2} v^{3}$ \\
\hline$g \rightarrow\left(c \bar{c}\left[{ }^{3} S_{1}^{(1)}\right]\right)$ & $\mathscr{O}\left(\alpha_{s}^{3}\right)$ & $\sim v^{3}$ & $\sim \alpha_{s}^{3} v^{3}$ \\
\hline$g \rightarrow\left(c \bar{c}\left[{ }^{3} S_{1}^{(8)}\right]\right)$ & $\mathscr{O}\left(\alpha_{s}^{1}\right)$ & $\sim v^{7}$ & $\sim \alpha_{s}^{1} v^{7}$ \\
\hline$g \rightarrow\left(c \bar{c}\left[{ }^{1} S_{0}^{(8)}\right]\right)$ & $\mathscr{O}\left(\alpha_{s}^{2}\right)$ & $\sim v^{7}$ & $\sim \alpha_{s}^{2} v^{7}$ \\
\hline$g \rightarrow\left(c \bar{c}\left[{ }^{3} P_{J}^{(8)}\right]\right)$ & $\mathscr{O}\left(\alpha_{s}^{2}\right)$ & $\sim v^{7}$ & $\sim \alpha_{s}^{2} v^{7}$ \\
\hline
\end{tabular}

Table 1: Leading contributions to the $J / \psi$ charm quark and gluon fragmentation function.

dominate the cross section since they scale with $\left(2 m_{c} / p_{T}\right)^{2}$ compared to other processes. Thus, in this region of transverse momentum, further reorganization of the perturbative expansion is required in order to properly account for all relevant fragmentation processes.

At large transverse momentum of the quarkonium state where fragmentation processes dominate, the cross section $d \sigma(a+b \rightarrow \mathscr{Q}+X)$ can be approximated using the leading power (LP) factorization [8] involving the fragmentation functions, $D_{i / \mathscr{Q}}$,

$$
\frac{d \sigma(a+b \rightarrow \mathscr{Q}+X)}{d x}=\sum_{i} \int_{x}^{1} \frac{d z}{z} d \sigma^{i}(z, \mu) D_{i / \mathscr{Q}}\left(\frac{x}{z}, \mu\right)+\mathscr{O}\left(m_{\mathscr{Q}}^{2} / p_{T}^{2}\right)
$$

where $d \sigma^{i}$ is the partonic cross section describing the hard process $a+b \rightarrow i+X$ and can be calculated in perturbation theory ${ }^{1}$. For the case of quarkonium the fragmentation functions can be further factorized using the NRQCD factorization conjecture [1],

$$
D_{i / \mathscr{Q}}(z, \mu)=\sum_{n} d_{i}^{n}(z)\left\langle\mathscr{O}_{\mathscr{Q}}^{n}\right\rangle
$$

In this work we are considering the five leading (through fragmentation) production mechanisms of $J / \psi$ shown in Table 1 . We isolate on $J / \psi$ fragmentation processes by requiring the charmonium to be produced within a jet and carrying significant energy of the jet. It was proposed in [9] that additional measurements on the jet substructure can be used in order to improve the discriminating power between the leading production mechanisms. In practice, we only need to discriminate between the various color-octet mechanisms since the color-singlet LDME can be constrained by potential models and more accurately extracted from the width $\Gamma\left[J / \psi \rightarrow \ell^{+} \ell^{-}\right]$.

\section{Review of fragmenting jet functions}

Fragmenting jet functions, $\mathscr{G}_{i / h}^{\text {alg. }}(z, \omega R)[10]$ describe the collinear splitting $i \rightarrow h+X$ within a jet and depend on the hadron's energy fraction $z=E_{h} / E_{J}$, the jet algorithm, and the (along the jet

\footnotetext{
${ }^{1}$ If this describes a hadronic collision then non-perturbative input from the corresponding parton distribution functions is required.
} 
axis) boost invariant combination $\omega R \equiv 2 E_{J} \tan (R / 2)$, where $E_{J}$ is the jet energy and $R$ is the jet size parameter. Furthermore fragmenting jet functions could depend on jet substructure observables if such observable is measured in the process considered. For example, $\mathscr{G}_{i / h}^{\text {alg. }}\left(z, \omega R, m_{J}\right)$ describe the fragmentation of an identified hadron, $h$, with energy $z E_{J}$, within a jet with energy $E_{J}$ for which the jet mass, $m_{J}$, is measured (see Refs. [11, 12]). Although FJFs are fundamentally non-perturbative objects, in the collinear limit can be written as a convolution of perturbative calculable matching coefficients, $C_{i / j}^{\mathrm{alg}}$, and the collinear fragmentation functions,

$$
\mathscr{G}_{i / h}^{\text {alg. }}\left(z, \omega R ; \mu_{J}\right)=\sum_{j} \int_{z}^{1} \frac{d x}{x} C_{i / j}^{\text {alg. }}\left(x, \omega R ; \mu_{J}\right) D_{j / h}\left(\frac{z}{x} ; \mu_{J}\right)+\mathscr{O}\left(\frac{\Lambda_{Q C D}^{2}}{\mu_{J}^{2}}\right),
$$

where the coefficients $C_{i / j}^{\text {alg. }}\left(x, \omega R ; \mu_{J}\right)$ contain all the jet algorithm and substructure measurement dependence of the FJF. The collinear fragmentation functions, $D_{i / h}(z)$, are evaluated at the jet scale, $\mu_{J}=\omega R$, and convolved with the matching coefficients evaluated at the same scale. The FJF is then evolved to an arbitrary factorization scale, $\mu$, according to the following renormalization group equation (RGE),

$$
\mu \frac{d}{d \mu} \mathscr{G}_{i / h}^{\text {alg. }}(z, \omega R ; \mu)=\gamma_{J}(\mu, \omega R) \mathscr{G}_{i / h}^{\text {alg. }}(z, \omega R ; \mu)
$$

where $\gamma_{J}(\mu, \omega R)$ is the jet function anomalous dimension (see Ref. [13]). In the case where jet substructure is measured then the RHS of the above equation involves a convolution between the anomalous dimension and the FJF.

In Ref. [15] for describing the production of quarkonium within jets in hadronic collisions we convolve the FJF with the corresponding hard scattering process evaluated using the monte-carlo software MadGraph [14],

$$
\frac{d \sigma}{d z}(p p \rightarrow \operatorname{jet}(J / \psi)+X) \sim \sum_{i=c, \bar{c}, g} \int d E \frac{d \sigma^{i}}{d E} \times \mathscr{G}_{i / \mathscr{Q}}^{\text {alg. }}(z, \omega R) .
$$

In Figure 1 we present the semi-analytical calculation (red band) using the above equation for proton-proton collisions at $\sqrt{s}=13 \mathrm{GeV}$ and compare against experimental data from $\mathrm{LHCb}$ [16]. We illustrate our results for three different sets of LDME. For the same sets of LDME we present the results using a modification of the simulation package PYTHIA [17, 18], called GFIP [12] (grey band), for which PYTHIA 8 is used for the partonic shower of the hard process and the resulted partonic distributions are then convolved to the quarkonium fragmentation functions. The values of LDME we used are: (left) the NLO global fit to the worlds data [4], (middle) fit at high $p_{T}$ where the channels $g \rightarrow c \bar{c}\left[{ }^{3} S_{1}^{(8)}\right]$ and $g \rightarrow c \bar{c}\left[{ }^{3} P_{J}^{(8)}\right]$ are tuned to cancel against each other [19], and (right) fit at high $p_{T}$ using the leading power factorization matched at NLO [20]. Compared against experimental data, our results show that the calculated energy fraction spectra of $J / \psi$ agree better with the data for some fits of the LDME compared to others. Although these results are encouraging, the fits from Refs. [19] and [20], which seem to agree best with the experimental data, have large uncertainties $(\sim 100 \%)$ for the $\left[{ }^{3} S_{1}^{(8)}\right]$ and $\left[{ }^{3} P_{J}^{(8)}\right]$ LDME.

In the next section we show that additional measurements on the $J / \psi$ can provide sufficient discriminating power between the five leading production mechanisms and thus can be used for fitting the values of the corresponding LDME. Particularly we propose a measurement of the quarkonium transverse momentum with respect to jet axis and we review the theoretical tools developed 


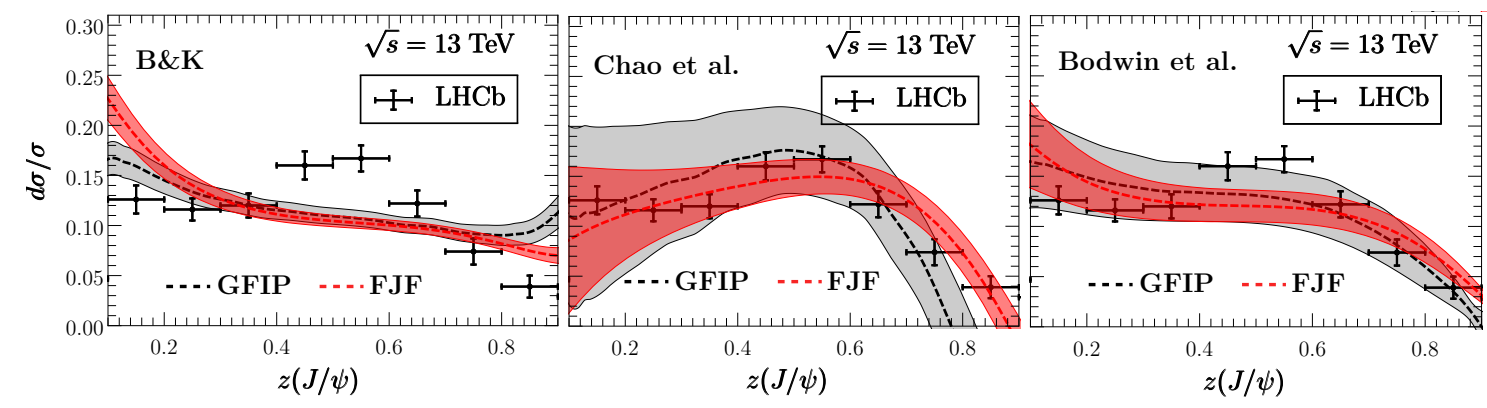

Figure 1: Predicted $z(J / \psi)$ distribution using GFIP (gray) and FJF (red) for the three choices of LDME as described in the text and the LHCb measurements of $z(J / \psi)$ [16]. Figure taken from [15]

in Ref. [23] necessary for this calculation. We show that, at large values of the energy ratio, such measurement can provide the desirable discriminating power between the color-octet production mechanisms.

\section{Transverse momentum dependent fragmenting jet functions}

Similarly to the FJF reviewed in the previous section, TMD-FJF describe the within jet fragmentation of an identified hadron, for which the energy fraction and the transverse momentum is measured. In the definition we consider here transverse momentum is measured with respect to the jet axis. Our formalism is consistent with the factorization of $e^{+} e^{-} \rightarrow \mathrm{N}$-jets exclusive processes in SCET described in Ref [13]. For alternative definitions of similar observables see Refs. [21, 22]. The operator definition of TMD-FJF is the following,

$$
\mathscr{G}_{q / h}\left(\overrightarrow{\mathrm{p}}_{\perp}, z, \mu\right)=\frac{1}{z} \sum_{X} \frac{1}{2 N_{c}} \delta\left(p_{X h ; r}^{-}\right) \delta^{(2)}\left(\overrightarrow{\mathrm{p}}_{\perp}+\overrightarrow{\mathrm{p}}_{\perp}^{X}\right) \operatorname{Tr}\left[\frac{\hbar}{2}\left\langle 0\left|\delta_{\omega, \overline{\mathscr{P}}} \chi_{n}^{(0)}(0)\right| X h\right\rangle\left\langle X h\left|\bar{\chi}_{n}^{(0)}(0)\right| 0\right\rangle\right],
$$

where the state $|X h\rangle$ corresponds to the a final state of collinear particles within a jet. In the collinear limit (i.e. $\overrightarrow{\mathrm{p}}_{\perp} \gg \Lambda_{Q C D}$ ) we could factorize this result into a convolution of perturbative short distance coefficients and the collinear fragmentation functions. The short distance coefficients are then further factorized into a collinear and collinear-soft (c-soft) contributions,

$$
\mathscr{G}_{i / h}\left(\overrightarrow{\mathrm{p}}_{\perp}, z ; \mu\right)=\int_{z}^{1} \frac{d x}{x} C_{i / j}\left(\overrightarrow{\mathrm{p}}_{\perp}, x ; \mu\right) D_{j / h}\left(\frac{z}{x} ; \mu\right)+\mathscr{O}\left(\frac{\Lambda_{Q C D}^{2}}{p_{\perp}^{2}}\right),
$$

where

$$
\left.C_{i / j}\left(\overrightarrow{\mathrm{p}}_{\perp}, z ; \mu\right)=N_{c} \times \int d^{2} \overrightarrow{\mathrm{p}}_{\perp}^{\prime} J_{i / j}\left(\overrightarrow{\mathrm{p}}_{\perp}-\overrightarrow{\mathrm{p}}_{\perp}^{\prime}, z ; \mu, v\right) S_{C}\left(\overrightarrow{\mathrm{p}}_{\perp}^{\prime} ; \mu, v\right)\right],
$$

with $J_{j} / i$ and $S_{C}$ the collinear and c-soft functions respectively. The scaling of the momentum of collinear and c-soft modes is,

$$
\begin{aligned}
\text { collinear-soft : } & p_{c s}^{\mu} \sim \omega(\lambda R, \lambda / R, \lambda), \quad \lambda=p_{\perp} / \omega \\
\text { collinear : } & p_{n}^{\mu} \sim \omega\left(\lambda^{2}, 1, \lambda\right) .
\end{aligned}
$$

The factorization of the c-soft and collinear modes is achieved through a BPS field redefinition 

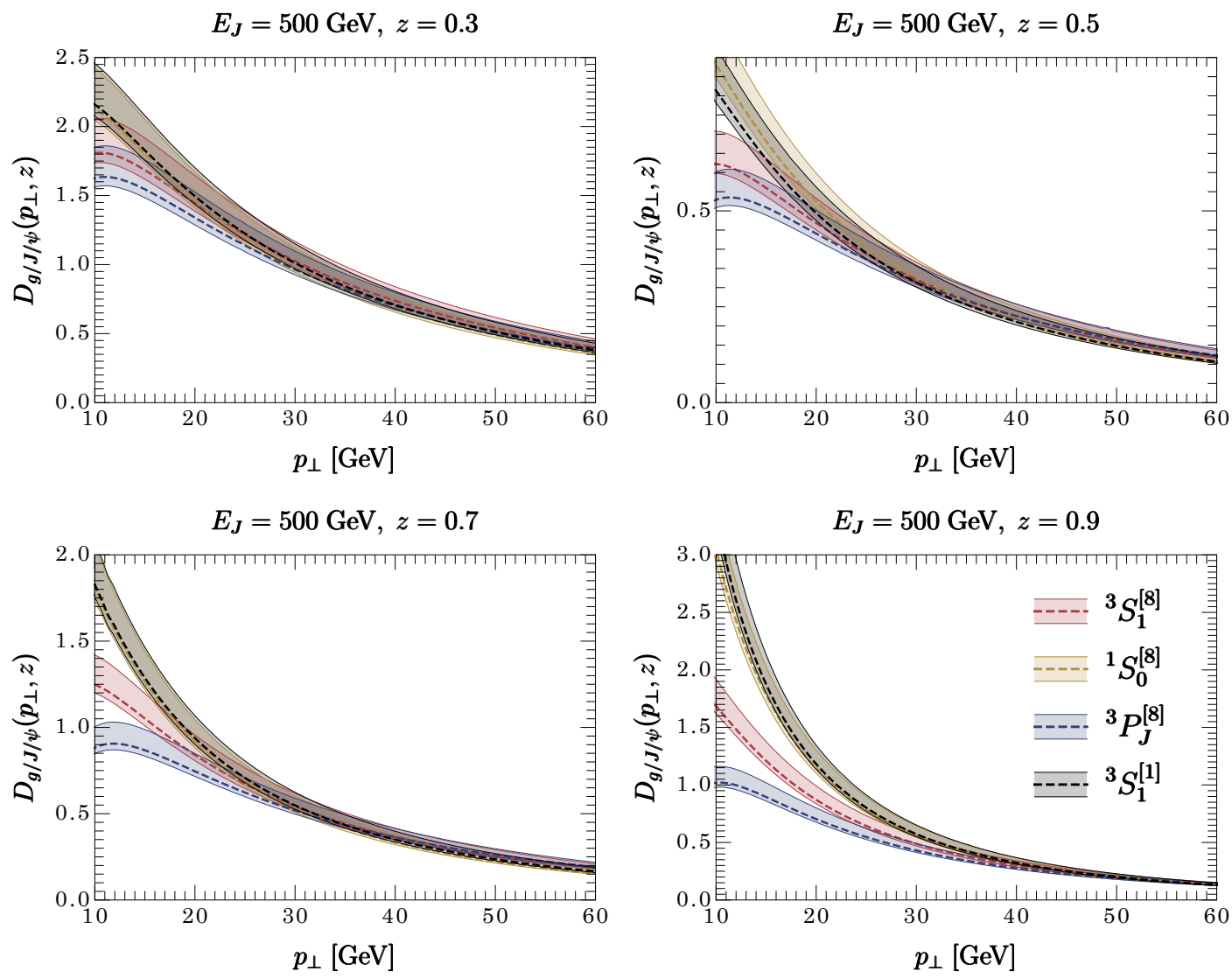

Figure 2: Transverse momentum spectrum of $J / \psi$ with in jets with energy $500 \mathrm{GeV}$, using anti- $k_{T}$ algorithm with jet size $R=0.6$ for $z_{J / \psi}=0.3,0.5,0.7$, and 0.9 .

which decouples the two modes at the level of the Lagrangian. The collinear and c-soft functions suffer from rapidity divergences, which is common for functions related to similar observables. These divergences appear due to the fact that collinear and c-soft modes (for measured transverse momentum) have the same virtuality and hence different regulator (other than dimensional regulator) is required to break the degeneracy. In this work we choose to use the rapidity regulator introduced and developed in Refs. [24, 25]. In the calculation of the relevant diagrams the rapidity divergences manifest as poles in the regulator parameter. The removal of these divergences is done at the stage of renormalization. As explained in Refs. [24, 25] this process allows us to systematically derive and solve rapidity RGEs which resum (in this case) large logarithms of $p_{\perp} /(\omega R)$. Additionally the solution of the virtuality RGE resum logarithms of the ratio $p_{\perp} / \mu_{H}$, where $\mu_{H} \sim \omega$ is the hard scale of the problem. It should be noted that the rapidity divergences cancel in the convolution of the collinear and c-soft term and thus, the TMD-FJF is independent of the rapidity scale, $v$. Further details of the factorization and resummation process are shown Ref. [23].

The resummation and convolution of the various terms in the factorization is performed in the Fourier space where a profile function is used to avoid the Landau pole at large values of the impact parameter, $b$. Traditionally a non-perturbative model for the anomalous dimensions is used 
for describing the spectrum at $p_{\perp} \sim \Lambda_{Q C D}$. In our analysis, no non-perturbative model is required since we are interested in the $p_{\perp}$ regime where non-perturbative effects are highly suppressed.

Here the formalism of TMD-FJF is applied for quarkonium production within jets and the transverse momentum distributions for fixed values of the energy fraction are presented in Figure 2. We focus on the gluon fragmentation processes as shown in Table 1. The $c \rightarrow c \bar{c}\left[{ }^{3} S_{1}^{(1)}\right]$ production channel is associated with the same LDME as $g \rightarrow c \bar{c}\left[{ }^{3} S_{1}^{(1)}\right]$ channel which can be extracted from decay measurements, as explained in the first section. The four values of the energy fraction shown here are $z=0.3,0.5,0.7$, and 0.9 . In each plot the distributions for the four production mechanisms are normalized to have approximately the same hight at the last bin, $50<p_{\perp}<60 \mathrm{GeV}$. We note that the color-singlet gluon fragmentation give almost identical spectrum as the ${ }^{1} S_{0}^{(8)}$ channel for all values of $z$, yet our result clearly show that the color-octet production mechanisms give distinct distributions for large values of energy fraction. Thus, focusing on the high $z$ region could provide sufficient discriminating power for extracting the corresponding LDME.

It is important to study if the same feature remains true in the total cross section for the case of hadronic collisions when one needs to consider contamination of jets from initial state radiation and multiparton interactions, collectively refer to as underlying event (UE). There has been recent developments towards eliminating the contamination in studies of jet substructure with the use of jet grooming (on jet grooming see for example Refs. [26, 27, 28, 29]), showing that for groomed jets, the jet shape observables are minimally effected by the UE [30, 31]. Extending this work to groomed jets will be important step towards implementing the formalism of TMD-FJF for hadronic collisions.

\section{Acknowledgments}

I would like to thank my collaborators: R. Bain, L. Dai, A. Leibovich, and T. Mehen. During the completion of this work YM was supported in part by the Director, Office of Science, Office of Nuclear Physics, of the U.S. Department of Energy under grant numbers DE-FG02- 05ER41368.

\section{References}

[1] G. T. Bodwin, E. Braaten and G. P. Lepage, Phys. Rev. D 51 (1995) 1125 Erratum: [Phys. Rev. D 55 (1997) 5853] doi:10.1103/PhysRevD.55.5853, 10.1103/PhysRevD.51.1125 [hep-ph/9407339].

[2] G. P. Lepage, L. Magnea, C. Nakhleh, U. Magnea and K. Hornbostel, Phys. Rev. D 46 (1992) 4052 doi:10.1103/PhysRevD.46.4052 [hep-lat/9205007].

[3] W. E. Caswell and G. P. Lepage, Phys. Lett. 167B (1986) 437. doi:10.1016/0370-2693(86)91297-9

[4] M. Butenschoen and B. A. Kniehl, Phys. Rev. D 84 (2011) 051501 doi:10.1103/PhysRevD.84.051501 [arXiv:1105.0820 [hep-ph]].

[5] R. Aaij et al. [LHCb Collaboration], Eur. Phys. J. C 73 (2013) no.11, 2631 doi:10.1140/epjc/s10052-013-2631-3 [arXiv:1307.6379 [hep-ex]].

[6] S. Chatrchyan et al. [CMS Collaboration], Phys. Lett. B 727 (2013) 381 doi:10.1016/j.physletb.2013.10.055 [arXiv:1307.6070 [hep-ex]].

[7] B. Abelev et al. [ALICE Collaboration], Phys. Rev. Lett. 108 (2012) 082001 doi:10.1103/PhysRevLett.108.082001 [arXiv:1111.1630 [hep-ex]]. 
[8] J. C. Collins and D. E. Soper, Nucl. Phys. B 194 (1982) 445. doi:10.1016/0550-3213(82)90021-9

[9] M. Baumgart, A. K. Leibovich, T. Mehen and I. Z. Rothstein, JHEP 1411 (2014) 003 doi:10.1007/JHEP11(2014)003 [arXiv:1406.2295 [hep-ph]].

[10] M. Procura and I. W. Stewart, Phys. Rev. D 81, 074009 (2010) Erratum: [Phys. Rev. D 83, 039902 (2011)] doi:10.1103/PhysRevD.81.074009, 10.1103/PhysRevD.83.039902 [arXiv:0911.4980 [hep-ph]].

[11] A. Jain, M. Procura and W. J. Waalewijn, JHEP 1105, 035 (2011) doi:10.1007/JHEP05(2011)035 [arXiv:1101.4953 [hep-ph]].

[12] R. Bain, L. Dai, A. Hornig, A. K. Leibovich, Y. Makris and T. Mehen, JHEP 1606 (2016) 121 doi:10.1007/JHEP06(2016)121 [arXiv:1603.06981 [hep-ph]].

[13] S. D. Ellis, C. K. Vermilion, J. R. Walsh, A. Hornig and C. Lee, JHEP 1011 (2010) 101 doi:10.1007/JHEP11(2010)101 [arXiv:1001.0014 [hep-ph]].

[14] J. Alwall et al., JHEP 1407 (2014) 079 doi:10.1007/JHEP07(2014)079 [arXiv:1405.0301 [hep-ph]].

[15] R. Bain, L. Dai, A. Leibovich, Y. Makris and T. Mehen, Phys. Rev. Lett. 119 (2017) no.3, 032002 doi:10.1103/PhysRevLett.119.032002 [arXiv:1702.05525 [hep-ph]].

[16] R. Aaij et al. [LHCb Collaboration], Phys. Rev. Lett. 118 (2017) no.19, 192001 doi:10.1103/PhysRevLett.118.192001 [arXiv:1701.05116 [hep-ex]].

[17] T. Sjostrand, S. Mrenna and P. Z. Skands, JHEP 0605 (2006) 026 doi:10.1088/1126-6708/2006/05/026 [hep-ph/0603175].

[18] T. Sjostrand, S. Mrenna and P. Z. Skands, Comput. Phys. Commun. 178 (2008) 852 doi:10.1016/j.cpc.2008.01.036 [arXiv:0710.3820 [hep-ph]].

[19] K. T. Chao, Y. Q. Ma, H. S. Shao, K. Wang and Y. J. Zhang, Phys. Rev. Lett. 108 (2012) 242004 doi:10.1103/PhysRevLett.108.242004 [arXiv:1201.2675 [hep-ph]].

[20] G. T. Bodwin, H. S. Chung, U. R. Kim and J. Lee, Phys. Rev. Lett. 113 (2014) no.2, 022001 doi:10.1103/PhysRevLett.113.022001 [arXiv:1403.3612 [hep-ph]].

[21] D. Neill, I. Scimemi and W. J. Waalewijn, JHEP 1704 (2017) 020 doi:10.1007/JHEP04(2017)020 [arXiv:1612.04817 [hep-ph]].

[22] Z. B. Kang, X. Liu, F. Ringer and H. Xing, arXiv:1705.08443 [hep-ph].

[23] R. Bain, Y. Makris and T. Mehen, JHEP 1611 (2016) 144 doi:10.1007/JHEP11(2016)144 [arXiv:1610.06508 [hep-ph]].

[24] J. Y. Chiu, A. Jain, D. Neill and I. Z. Rothstein, JHEP 1205 (2012) 084 doi:10.1007/JHEP05(2012)084 [arXiv:1202.0814 [hep-ph]].

[25] J. y. Chiu, A. Jain, D. Neill and I. Z. Rothstein, Phys. Rev. Lett. 108 (2012) 151601 doi:10.1103/PhysRevLett.108.151601 [arXiv:1104.0881 [hep-ph]].

[26] M. Cacciari, G. P. Salam and G. Soyez, JHEP 0804 (2008) 005 doi:10.1088/1126-6708/2008/04/005 [arXiv:0802.1188 [hep-ph]].

[27] D. Krohn, J. Thaler and L. T. Wang, JHEP 1002 (2010) 084 doi:10.1007/JHEP02(2010)084 [arXiv:0912.1342 [hep-ph]].

[28] D. Bertolini, P. Harris, M. Low and N. Tran, JHEP 1410 (2014) 059 doi:10.1007/JHEP10(2014)059 [arXiv:1407.6013 [hep-ph]]. 
[29] C. Frye, A. J. Larkoski, M. D. Schwartz and K. Yan, JHEP 1607 (2016) 064 doi:10.1007/JHEP07(2016)064 [arXiv:1603.09338 [hep-ph]].

[30] A. J. Larkoski, I. Moult and D. Neill, arXiv:1710.00014 [hep-ph].

[31] I. Moult, B. Nachman and D. Neill, arXiv:1710.06859 [hep-ph]. 